able criticisms and suggestions, I am exceedingly thankful to Robert MacArthur, Egbert Leigh, Christopher Smith, Howard Howland, Elizabeth Horn, John Bonner, Gordon Orians, and David Horn. Thomas Frazzetta, Richard Horn, Daniel Janzen, Otto Solbrig, and Carl Gans read and commented on parts of the manuscript.

The book is dedicated to my father, Henry E. Horn, who introduced me to forests when I was four years old.

\title{
Preface to the Second Printing (1976)
}

Since 1971 there have been several developments in the literature and in my own thinking that would make this book different were I to write it now. The outline, arguments, and results would be the same, but I would change some of the following details.

Chapters $4^{-6}$ give the impression that the technical development of the theory rests heavily on the assumption that leaves are randomly distributed disks. However, Equations $5.8,5.10$, and 5.13 could be derived with even simpler notation from an arbitrary index of leaf area per unit of ground area, with leaves distributed into independent layers, within each of which the leaves are evenly dispersed. The models are not restricted to a peculiar leaf shape or to a particular vertical distribution of leaves.

I have recently described the difference between monolayered and multilayered trees more graphically (1975. Scientific American 232 (5): 9o-98). The essential difference is less between different vertical distributions of branches, than between a tree with its leaves in a continuous shell about the peripheral twigs and a tree with leaves scattered loosely throughout its volume (cf. page 63). In the same article, I address the question of why individual trees tend to be either monolayered or multilayered, with only limited 


\section{PREFACE TO SECOND PRINTING}

flexibility between these extremes. Whitney (1976. Bull. Torrey Bot. Club, in press) has related leaf distribution to branching structure. I have expanded the discussion in Chapter 8 of patterns of productivity, stability, and diversity in succession (1974. Ann. Rev. Ecol. Syst. 5: 25-37).

In Chapters 2 and $6, I$ leave the impression that special equipment is needed to measure the proportion of light that directly penetrates a branch or a tree. However, for measuring "layers" in a tree, surprising accuracy can be attained by rolling up a piece of paper into a tube, sighting the appropriate branch or tree, adjusting the width of the tube or its distance from the eye until only the section to be measured is seen, and guessing the proportion of sky that is unobscured. Accuracy in the range between 0.1 and 0.9 comes quickly with experience. Beyond this range even a light meter is often inaccurate. Accuracy is not critical however. Similar errors affect the measurement of both branch and tree. When several Princeton undergraduates have measured the same tree, their raw data seem appallingly disparate, but the ratio of logarithms of each individual's measurements, that is the number of "layers," shows little variation between observers. The errors that persist involve very sparsely foliated branches on very shady trees. These are multilayers, for which compulsive accuracy is fatuous because the photosynthetic difference between 2 and 5 layers is about the same as that between 5 layers and an infinite number (page 78 ).

Monsi, Uchijima, and Oikawa (1973. Ann. Rev. Ecol. Syst. 4: 301-327) review more accurate and perforce less general studies of the effect of canopy structure on photosynthesis. The cyclic persistence of Beech and Sugar Maple, inferred on pages $35-37$, has been explored by Forcier (1975. Science 189: 8o8-810), who interprets their presence with Yellow Birch (Betula alleghaniensis) in central New Hamp- 
PREFACE TO SECOND PRINTING

shire as a "climax microsuccession." The inferred allelopathic effect of Sassafras (pages 40-41) has been documented and explored by Gant and Clebsch (1975. Ecology 56: 604615). Anderson and Miller (1974. J. Appl. Ecol. 11: 691697) discuss the penumbral effect of leaves (pages 46-47) and sunflecks in the context of some striking pictures. Dahl (1973. Marine Biol. 23: 239-249) has invented geometric models to describe corals in a way that is more appropriate than the suggestion on page 129 .

An approach analogous to mine can be applied to the stratification of trees themselves in storied forests, as Smith (1973. Am. Naturalist 107: 671-683) has done verbally, and $\mathrm{J}$. W. Terborgh has done analytically in a lecture that he promises to publish soon. Terborgh's ideas will be reviewed in a forthcoming book by F. Hallé, R.A.A. Oldeman, and P. B. Tomlinson (in preparation. Tree Architecture in Tropical Forest Ecosystems. Springer Verlag, New York \& Heidelberg). This book will also discuss developmental patterns that may set limitations on the shapes and leaf distributions of trees of a given species. 